\title{
Global fluid phase behavior in binary mixtures of rodlike and platelike molecules
}

\author{
Szabolcs Varga, Amparo Galindo, and George Jackson \\ Department of Chemical Engineering and Chemical Technology, Imperial College of Science, \\ Technology and Medicine, Prince Consort Road, London SW7 2BY, United Kingdom
}

(Received 21 May 2002; accepted 24 July 2002)

The phase behavior of a liquid-crystal forming model colloidal system containing hard rodlike and platelike particles is studied using the Parsons-Lee scaling [J. D. Parsons, Phys. Rev. A 19, 1225 (1979); S. D. Lee, J. Chem. Phys. 87, 4972 (1987)] of the Onsager theory. The rod and plate molecules are both modeled as hard cylinders. All of the mixtures considered correspond to cases in which the volume of the plate is orders of magnitude larger that the volume of the rod, so that an equivalence can be made where the plates are colloidal particles while the rods play the role of a depleting agent. A combined analysis of the isotropic-nematic bifurcation transition and spinodal demixing is carried out to determine the geometrical requirements for the stabilization of a demixing transition involving two isotropic phases. Global phase diagrams are presented in which the boundaries of isotropic phase demixing are indicated as functions of the molecular parameters. Using a parameter $z$ which corresponds to the product of the rod and plate aspect ratios, it is shown that the isotropic phase is unstable relative to a demixed state for a wide range of molecular parameters of the constituting particles due to the large excluded volume associated with the mixing of the unlike particles. However, the stability analysis indicates that for certain aspect ratios, the isotropic-nematic phase equilibria always preempts the demixing of the isotropic phase, irrespective of the diameters of the particles. When isotropic-isotropic demixing is found, there is an upper bound at large size ratios (Asakura and Oosawa limit), and a lower bound at small size ratios (Onsager limit) beyond which the system exhibits a miscible isotropic phase. It is very gratifying to find both of these limits within a single theoretical framework. We test the validity of the stability analysis proposed by calculating a number of phase diagrams of the mixture for selected molecular parameters. As the hard rod particles promote an effective attractive interaction between the hard-plate colloidal particles, the isotropic-isotropic demixing usually takes place between two rod-rich fluids. As far as the isotropic-nematic transition is concerned, a stabilization as well as a destabilization of the nematic phase relative to the isotropic phase is seen for varying rod-plate size ratios. Moreover, isotropic-nematic azeotropes and re-entrant phenomena are also observed in most of the mixtures studied. We draw comparisons between the predicted regions of stability for isotropic demixing and recent experimental observations. (c) 2002 American Institute of Physics.

[DOI: $10.1063 / 1.1507112]$

\section{INTRODUCTION}

Binary mixtures of hard particles can exhibit a surprisingly complex phase behavior. For large size differences, attractive depletion interactions can be induced which may result in phase separations. Phase transitions in the liquid phase of multicomponent mixtures of hard spherical particles have been the focus of a great deal of attention (e.g., see the recent work $^{1,2}$ ), but these systems of spherical particles are not the focus of our current work. On the other hand, it is known that hard nonspherical particles can exhibit liquidcrystalline phase behavior including nematic (or discotic) and smectic (or columnar) phases. In these mixtures, a competition between demixing and ordering determines the stability of the many phases. A knowledge of the phase behavior in such systems is of prime practical importance in understanding colloidal products (foods, cosmetics, paints, blood,...), and it can give an insight into the fundamentals of phase transitions.
After the discovery of a first-order isotropic-nematic phase transition in a suspension of rodlike particles, ${ }^{3}$ many other colloidal systems of such particles have been found to give rise to ordered fluid phases including layering into smectics (see, for example, Ref. 4). Equivalent types of phase transitions have been confirmed more recently in discotic colloidal systems. Lekkerkerker and co-workers have observed isotropic-nematic ${ }^{5}$ and nematic-columnar ${ }^{6}$ phase transitions in sterically stabilized colloidal suspensions of gibbsite platelets. The colloidal system of Lekkerkerker and co-workers is highly polydisperse, but in a separate study by Brown et al., ${ }^{7}$ monodisperse platelike particles of nickel(II) hydroxide have also been shown to exhibit structures of higher order than the nematic phase. Monte Carlo simulation studies $^{8,9}$ of the phase behavior of hard disklike particles confirm that such systems can exhibit nematic, columnar, and even cubatic phases, although the latter have not been identified experimentally. 
It is well documented ${ }^{10}$ that the interactions between colloidal particles can be tuned by the addition of nonadsorbing polymers to a suspension of the hard particles. The polymer molecules can induce an effective attractive interaction between the colloidal particles as they maximize their free volume by promoting the clustering of the large colloidal particles. This is the so-called depletion effect. The range and strength of the depletion interaction can be varied by changing the size and concentration of the polymer component to such an extent that it can give rise to the phase separation between two colloid-rich isotropic phases. This separation provides a useful means of purifying polydisperse colloidal mixtures. ${ }^{11}$ The effect of the depletion interactions in mixtures of rodlike colloidal particles has been investigated experimentally using a flexible nonadsorbing polymer; demixing between isotropic as well as nematic phases has been observed. ${ }^{12,13}$ A mixture of spherical colloidal particles with a rodlike polymer component has also been studied. ${ }^{14}$ The phase equilibria can become quite complex; for example, three and four phase coexistence of isotropic, nematic, and columnar phases has been observed experimentally in a mixture of platelike colloidal particles and nonadsorbing polymers. ${ }^{11}$ The overall conclusion of this body of work is that the depleting agent must be considerably smaller than the colloidal component in order to induce isotropicisotropic phase separation. Although the minimum size ratio needed to induce the demixing transition has been extensively studied, ${ }^{15-19}$ little is known about its upper limit. In a recent investigation of the phase behavior of a rod-plate binary mixture with particle volume ratios of 20/1 isotropic demixing was not seen. ${ }^{20}$ Furthermore, mixtures of plateand rodlike particles may give rise to stable biaxial phases as suggested by Alben. ${ }^{21}$ The possibility of finding a biaxial nematic phase in such systems has also been the subject of detailed investigation in recent years, ${ }^{22-25}$ but the findings remain inconclusive.

Theoretical methods can be used to explore the phase behavior of these complex mixtures by continuous changes of the molecular parameters of the components, so that colloid-polymer mixtures and colloid-colloid systems can be examined within the same framework. In this work we examine the global phase behavior of plate-rod binary mixtures in which the plate plays the role of the colloidal particle, while the rod, of much smaller volume, induces an attractive depletion interaction between the plates. We are particularly interested in determining the molecular requirements for which isotropic-isotropic demixing is observed. In future work we shall be considering symmetrical plate-rod mixtures concentrating on a study of the stability of the biaxial nematic phase. ${ }^{26}$

The theoretical treatment of nonspherical colloid suspensions dates back to the seminal work of Onsager, ${ }^{27}$ who showed that the isotropic-nematic phase transition of infinitely thin rodlike particles may be treated with a virial expansion of the free energy. Furthermore, if the colloidal particles are immersed in a so-called "good" solvent, the solvent can be assumed to have a negligible effect on the phase behavior of the colloidal component, so that only the difference in free energy between the solution and the sol- vent needs to be considered. Onsager showed that nematic ordering arises due to the subtle competition between two entropic contributions: an orientational entropy which favours disordered isotropic phases, and a packing entropy which maximizes the free volume available to the particles and favors orientational ordering. A drawback of the Onsager theory is that it is justified only for very long and thin particles as the virial expansion is described only up to second order in his approach. The extension of the Onsager theory to binary mixtures is straightforward, and it has been applied to a number of systems including rod-rod, ${ }^{28-31}$ plate-plate, ${ }^{32}$ and rod-plate ${ }^{22,23,25}$ binary mixtures. Isotropic-nematic, nematic-nematic, and isotropic-isotropic phase coexistence, as well as reentrant phenomena are commonly seen in these systems. The nematic-nematic transition usually occurs at very high pressure, hence involving highly ordered phases; one also finds upper and lower critical points which are associated with this demixing transition. It is interesting to note that upper critical pressures have been obtained for nematic demixing in mixtures of hard rodlike particles, ${ }^{29-31}$ while lower critical pressures are seen as the limit of nematic demixing in mixtures platelike particles. ${ }^{32}$ In hard rod-plate mixtures, the nematic directors of the rod-rich and plate-rich coexisting phases are always perpendicular to each other, so that high-pressure nematic-nematic critical points have not been observed. Furthermore, the large rod-plate excluded volume means that the region of nematic-nematic separation is very wide in composition. However, as we have mentioned earlier, the stability of a mixed biaxial nematic phase relative to the nematic-nematic separation is still a matter of debate (see Ref. 26, and references therein for details). The necessary conditions for isotropic-isotropic coexistence in mixtures of nonspherical hard particles have been previously investigated for a number of special cases. ${ }^{30,33-36}$ It is known, for example, that mixtures of rodlike particles of different lengths but equal diameters do not exhibit this phase separation, ${ }^{33}$ and that when the diameters are different, a critical volume ratio determines the stabilization of the transition. ${ }^{30,34,35}$ In the limit of very large size ratios, the smaller particles can be treated as ideal as far as the pure component interactions are concerned, although in the mixture the interactions between the small and large particles must be incorporated. This approximation is the well-known Asakura-Oosawa model, ${ }^{37}$ which has proved very useful in the description of colloid-polymer systems. ${ }^{10}$ Sear and Jackson ${ }^{15}$ have studied a colloid-polymer mixture in which the colloid particles are represented as hard rods, and the polymer molecules are modeled as rods of zero diameter; the colloid-colloid and colloid-polymer interactions were treated using the theory of Onsager in the Zwanzig ${ }^{38}$ approximation, and polymer-polymer interactions were treated as ideal. The resulting phase diagram exhibits a demixing transition in the isotropic phase for a polymer/colloid length ratio which is greater than about three. In subsequent work, Hemmer and Marthinsen ${ }^{18}$ determined the phase diagram for the mixture studied by Sear and Jackson, and examined a range of length ratios using the continuous version of the theory of Onsager (instead of the Zwanzing approximation ${ }^{38}$ ) incorporating the exact solution of the orientational distribu- 
tion functions. They find a critical length ratio of about onethird instead of three, but agree on the fact that larger polymer lengths enhance the stability of the isotropic-isotropic demixing region. It should be noted, however, that the Asakura-Oosawa model becomes unreliable when the rodlike polymer particles are very long as the assumption of ideality breaks down; such particles would be closer to the Onsager limit than to the ideal gas limit. Recent work has also been carried out on the transitions of platelike colloidpolymer systems. Using computer simulation and perturbation theory, Bates and Frenkel ${ }^{39}$ have examined the phase behavior in two colloidal systems of platelike molecules: one of these consists of a mixture of infinitely thin colloidal plates with penetrable (ideal) spheres playing the role of the polymer; the other involves infinitely thin colloidal plates and penetrable (ideal) rods. In both systems the isotropicisotropic demixing transition is strongly influenced by the ordering transitions, in the sense that a stabilization of the ordering transition may preempt the isotropic demixing. In the work of Bates and Frenkel, the Asakura-Oosawa model is assumed, and so isotropic demixing is always favored for the larger polymers. A fluid of platelike colloidal particles of finite thickness with penetrable (ideal) spheres has also been investigated, ${ }^{40}$ unfortunately, ordering was not included in the model, so it is difficult to determine the range of stability of the isotropic demixing transition. In a more recent work isotropic demixing has been confirmed in this system using computer simulations, in which ordered phases were taken into account. ${ }^{19}$

As we have already mentioned, it is generally concluded that within the Asakura-Oosawa model isotropic-isotropic separation is stabilized with increasing polymer sizes. The approach is however limited in terms of the size of the polymer, as it is considered as ideal in the model and hence has zero volume. It is interesting to investigate the importance of incorporating the excluded volume effects between polymer particles in studies of colloidal-polymer mixtures. We focus on a mixture of colloidal platelike particles with small rodlike particles which play the role of the "polymer" (depleting agent) taking into account plate-plate, rod-rod, and rod-plate interactions at the same level of approximation; one should note however, that in macromolecular systems the polymer molecules can penetrate to a certain extent so that in reality the polymer-polymer interactions are neither completely hard nor completely ideal. We use the continuous Onsager theory, together with the scaling of Parsons ${ }^{41}$ and Lee $^{42}$ (which corrects for the second virial truncation of the free energy expansion) to model the mixtures.

This paper is organized as follows: A short description of the Parsons-Lee theory for binary mixtures of hard-body fluids is given in Sec. II together with the expressions for the equilibrium orientational distribution functions and the details of the numerical phase equilibrium calculation. In Sec. III a general stability analysis is presented. The boundary values of the molecular parameters which delimit the regions of parameter-space with and without isotropic demixing are determined using a combined analysis of the isotropicnematic bifurcation (Sec. III A) and isotropic spinodal (Sec. III B) curves. The combined analysis is given in Sec. III C.

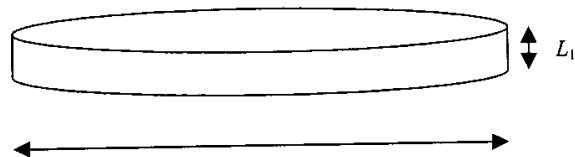

$D_{1}$

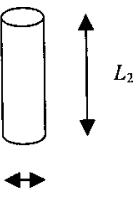

$D_{2}$
FIG. 1. The platelike and rodlike particles are modelled as hard cylinders. The platelike particles have a diameter $D_{1}$ and thickness $L_{1}$ with $L_{1} / D_{1}$ $<1$, while the rodlike particles have a diameter $D_{2}$ and length $L_{2}$ with $L_{2} / D_{2}>1$.

The molecular models used are introduced in Sec. IV, results are given in Sec. V, and we end with some general conclusions in Sec. VI.

\section{PARSONS-LEE THEORY}

We consider a binary mixture of hard cylinders, exploring the phase behavior of systems in which one of the components is thin and flat (i.e., platelike), and the other is long and thin (i.e., rodlike) (see Fig. 1). In order to study the mixture phase behavior we use the extension of the ParsonsLee theory for hard-body fluids presented by Camp and Allen. ${ }^{43,44}$ Parsons, ${ }^{41}$ and later Lee, ${ }^{42}$ independently proposed a scaling procedure to take into account the high virial terms which are neglected in the second virial theory of Onsager for the nematic phase. ${ }^{27}$ The Parsons-Lee approach, as that of Onsager, is suitable for studies of phase transitions between orientationally disordered and orientationally ordered fluid phases (i.e., isotropic-nematic transitions), but not for phases exhibiting higher degrees of order, such as smectic, columnar, or solid phases. While the theory of Onsager for rod particles is exact in the limit of infinite elongations,${ }^{27}$ it quickly breaks down for smaller aspect ratios. An advantage of the Parsons-Lee approach is that it provides an accurate representation of the phase behavior of anisotropic hard particles which are not necessarily of infinite aspect ratio.

In the Parsons-Lee approximation the Helmoltz free energy of the binary mixture is a functional of the orientational distribution functions $\left(f_{i}, i=1,2\right)$ of each component $i$ in the mixture, and is given by

$$
\begin{aligned}
\frac{\beta F}{N}= & \sum_{i=1}^{2} x_{i}\left(\ln \rho-1+\ln \frac{\mathrm{v}_{i}}{4 \pi}+\ln x_{i}+\sigma\left[f_{i}\right]\right) \\
& +\frac{\beta F_{\mathrm{ex}}^{\mathrm{hs}} B_{2}}{N 4 v_{\mathrm{hs}}},
\end{aligned}
$$

where $\beta=1 / k T$ ( $T$ is the temperature and $k$ is Boltzmann's constant), $\rho=N / V$ is the number density, $x_{i}$ is the mole fraction of component $i$, and $\mathrm{v}_{i}$ is the de Broglie volume which takes into account the translational and rotational contributions of the kinetic energy of each component. The sum corresponds to the ideal translational/rotational contribution, the ideal entropy of mixing, and the ideal orientational entropy. The orientational entropy is determined from the normalized equilibrium orientational distribution functions $\int f_{i}(\boldsymbol{\omega}) d \boldsymbol{\omega}$ $=1$ of each component as 


$$
\sigma\left[f_{i}\right]=\int f_{i}(\boldsymbol{\omega}) \ln \left(4 \pi f_{i}(\boldsymbol{\omega})\right) d \boldsymbol{\omega},
$$

where $\boldsymbol{\omega}$ is the orientational unit vector defined by a polar angle $(0<\theta<\pi)$ and an azimuthal angle $(0<\varphi<2 \pi)$. The last term in Eq. (1), which takes into account the repulsive interactions, is approximated according to the prescription of the Parsons-Lee theory: ${ }^{41,42} F_{\mathrm{ex}}^{\mathrm{hs}}$ and $v_{\mathrm{hs}}$ are the residual free energy and volume of an equivalent hard-sphere system, while the second virial coefficient $B_{2}$ is a mole fraction weighted sum of the like $B_{11}$ and $B_{22}$, and unlike $B_{12}$ second virial coefficients, so that

$$
B_{2}=x_{1}^{2} B_{11}+2 x_{1} x_{2} B_{12}+x_{2}^{2} B_{22} .
$$

In the case of hard particles, the second virial coefficient $B_{i j}$ can be obtained from an orientational average of the excluded volume $v_{i j}$ between molecules $i$ and $j$ as ${ }^{10}$

$$
B_{i j}=\frac{1}{2} \int v_{i j}\left(\boldsymbol{\omega}_{1}, \boldsymbol{\omega}_{2}\right) f_{i}\left(\boldsymbol{\omega}_{1}\right) f_{j}\left(\boldsymbol{\omega}_{2}\right) d \boldsymbol{\omega}_{1} d \boldsymbol{\omega}_{2} .
$$

At this stage it is necessary to specify the reference hardsphere system, i.e., $v_{\text {hs }}$. Following Camp and Allen, ${ }^{43}$ the total volume occupied by the particles is written in terms of a hard-sphere volume. The volume of the hard sphere $v_{\mathrm{hs}}$ is obtained as a mole-fraction weighted function of the volumes $v_{i}$ of each of the molecules,

$$
v_{\mathrm{hs}}=v_{1} x_{1}+v_{2} x_{2} \text {. }
$$

The residual free energy of the hard-sphere system which is used in the scaling of Parsons ${ }^{41}$ and Lee ${ }^{42}$ [see also Eq. (1)] is introduced using the expression of Carnahan and Starling 45 for a pure hard-sphere fluid,

$$
\frac{\beta F_{\mathrm{ex}}^{\mathrm{hs}}}{N}=\frac{4 \eta-3 \eta^{2}}{(1-\eta)^{2}},
$$

with the difference that in our present system the packing fraction $\eta$ is composition dependent. It is given by

$$
\eta=\eta^{*}\left(x_{1}+x_{2} \frac{v_{2}}{v_{1}}\right),
$$

where $\eta^{*}=N v_{1} / V$ is a reduced density.

Equations (4) and (5) are determined by the features of the molecules in the system (molecular size and shape), other than this, the free energy expressions for the mixture are general. If the molecular parameters are known so that the particle volumes and excluded volumes can be determined, expressions for the free energy can be obtained. It is important to mention however, that the scaling proposed would not be the most adequate for a hard-sphere mixture, and that more accurate expressions for the free energy of a mixture of hard spheres are well known. ${ }^{46,47}$ This approach is particularly suited to study isotropic-nematic transitions in mixtures involving nonspherical particles.

In order to study the phase equilibria in the Parsons-Lee approach, the equilibrium single particle orientational distribution functions $f_{i}(\boldsymbol{\omega})$ must also be determined. At equilibrium, the total free energy must be stationary with respect to variations of the equilibrium orientational distribution function, i.e.,

$$
\frac{\delta\left(\beta F / N+\lambda_{i}\left(1-\int f_{i}(\boldsymbol{\omega}) d \boldsymbol{\omega}\right)\right)}{\delta f_{i}(\boldsymbol{\omega})}=0,
$$

where $\lambda_{i}$ are Lagrange undetermined multipliers and the normalization condition $\int f_{i}(\boldsymbol{\omega}) d \boldsymbol{\omega}=1$ is also imposed. The resulting integral equations are

$$
\begin{aligned}
\ln \left(4 \pi f_{1}(\boldsymbol{\omega})\right)= & \lambda_{1}-\frac{f^{*}(\eta)}{4\left(x_{1} v_{1}+x_{2} v_{2}\right)} \\
& \times\left(x_{1} \int v_{11}\left(\boldsymbol{\omega} \boldsymbol{\omega}_{2}\right) f_{1}\left(\boldsymbol{\omega}_{2}\right) d \boldsymbol{\omega}_{2}\right. \\
& \left.+x_{2} \int v_{12}\left(\boldsymbol{\omega} \boldsymbol{\omega}_{2}\right) f_{2}\left(\boldsymbol{\omega}_{2}\right) d \boldsymbol{\omega}_{2}\right),
\end{aligned}
$$

and

$$
\begin{aligned}
\ln \left(4 \pi f_{2}(\boldsymbol{\omega})\right)= & \lambda_{2}-\frac{f^{*}(\eta)}{4\left(x_{1} v_{1}+x_{2} v_{2}\right)} \\
& \times\left(x_{2} \int v_{22}\left(\boldsymbol{\omega} \boldsymbol{\omega}_{2}\right) f_{2}\left(\boldsymbol{\omega}_{2}\right) d \boldsymbol{\omega}_{2}\right. \\
& \left.+x_{1} \int v_{12}\left(\boldsymbol{\omega} \boldsymbol{\omega}_{2}\right) f_{1}\left(\boldsymbol{\omega}_{2}\right) d \boldsymbol{\omega}_{2}\right) .
\end{aligned}
$$

The Lagrange multipliers can be eliminated using the normalization conditions of the orientational distribution functions to give the following self-consistent integral equations:

$$
f_{1}(\boldsymbol{\omega})=\frac{\exp \left(-\frac{f^{*}(\eta)}{4\left(x_{1} v_{1}+x_{2} v_{2}\right)}\left(x_{1} \int v_{11}\left(\boldsymbol{\omega} \boldsymbol{\omega}_{2}\right) f_{1}\left(\boldsymbol{\omega}_{2}\right) d \boldsymbol{\omega}_{2}+x_{2} \int v_{12}\left(\boldsymbol{\omega} \boldsymbol{\omega}_{2}\right) f_{2}\left(\boldsymbol{\omega}_{2}\right) d \boldsymbol{\omega}_{2}\right)\right)}{\int \exp \left(-\frac{f^{*}(\eta)}{4\left(x_{1} v_{1}+x_{2} v_{2}\right)}\left(x_{1} \int v_{11}\left(\boldsymbol{\omega}_{1} \boldsymbol{\omega}_{2}\right) f_{1}\left(\boldsymbol{\omega}_{2}\right) d \boldsymbol{\omega}_{2}+x_{2} \int v_{12}\left(\boldsymbol{\omega}_{1} \boldsymbol{\omega}_{2}\right) f_{2}\left(\boldsymbol{\omega}_{2}\right) d \boldsymbol{\omega}_{2}\right)\right) d \boldsymbol{\omega}_{1}},
$$

and

$$
f_{2}(\boldsymbol{\omega})=\frac{\exp \left(-\frac{f^{*}(\eta)}{4\left(x_{1} v_{1}+x_{2} v_{2}\right)}\left(x_{2} \int v_{22}\left(\boldsymbol{\omega} \boldsymbol{\omega}_{2}\right) f_{2}\left(\boldsymbol{\omega}_{2}\right) d \boldsymbol{\omega}_{2}+x_{1} \int v_{12}\left(\boldsymbol{\omega} \boldsymbol{\omega}_{2}\right) f_{1}\left(\boldsymbol{\omega}_{2}\right) d \boldsymbol{\omega}_{2}\right)\right)}{\int \exp \left(-\frac{f^{*}(\eta)}{4\left(x_{1} v_{1}+x_{2} v_{2}\right)}\left(x_{2} \int v_{22}\left(\boldsymbol{\omega}_{1} \boldsymbol{\omega}_{2}\right) f_{2}\left(\boldsymbol{\omega}_{2}\right) d \boldsymbol{\omega}_{2}+x_{1} \int v_{12}\left(\boldsymbol{\omega}_{1} \boldsymbol{\omega}_{2}\right) f_{1}\left(\boldsymbol{\omega}_{2}\right) d \boldsymbol{\omega}_{2}\right)\right) d \boldsymbol{\omega}_{1}},
$$


where we have used $f^{*}=\beta F_{\mathrm{hs}}^{\mathrm{ex}} / N$ [see Eq. (6)].

In order to avoid the uncertainties that arise when using the series-expansion methods, ${ }^{30}$ we solve the coupled integral equations [Eqs. (11) and (12)] numerically. Using a polar coordinate system, the unit vectors, excluded volumes and orientational distribution functions can be expressed as functions of the polar $\theta$ and azimuthal $\varphi$ angles. The integration over the orientational unit vector $\boldsymbol{\omega}$ is carried out by Simpson's quadrature. ${ }^{48}$ An interval $[0, \pi]$ divided into 160 equally spaced subintervals is used for $\theta$, while an interval $[0,2 \pi]$ divided into 320 subintervals is used for $\varphi$ to ensure an accurate representation of the integrals even if the orientational distribution functions are highly peaked. Since in this work we examine only orientationally disordered (isotropic) and axially symmetrically ordered (uniaxial nematic) phases (i.e., biaxial nematic, smectic, and solid phases are not taken into account) the orientational distribution functions can be defined to depend only on the polar angle. In the numerical procedure a highly-ordered axially symmetric orientational distribution functions are used as starting points for the iteration; we start with initial guesses for the two distribution functions using highly peaked Onsager trial functions one of which has a maximum in the direction of the director and the other which has a maximum in the plane perpendicular to the director. The procedure continues until $\max \left|f_{i, \text { new }}(\theta)-f_{i, \text { old }}(\theta)\right|<10^{-9} \quad(i=1,2)$. Once $f_{i}(\theta)$ is determined, the free energy is obtained, and the coexistence conditions (equality of pressure and chemical potentials) are solved using a Marquat-Levenberg algorithm; ${ }^{48}$ the numerical accuracy in the coexistence pressure $P=-[\partial F / \partial V]_{T, N}$ and chemical potentials $\mu_{i}=\left[\partial F / \partial N_{i}\right]_{T, V, N j \neq i}$ is chosen to be of at least 6 significant figures.

The extent of ordering in a phase is characterized with the uniaxial order parameter, which is defined as an average second Legendre polynomial $\left(P_{2}\right)$,

$$
S_{i}=\int P_{2}(\cos \theta) f_{i}(\theta) d \boldsymbol{\omega},
$$

where a value of $S_{i}>0$ indicates molecular alignment along the $z$ axis (direction of the nematic director) in a nematic phase, while $S_{i}<0$ means that alignment occurs in a direction normal to the nematic director (planar phase), and $S_{i}$ $=0$ corresponds to a disordered isotropic phase. In rod-plate mixtures these order parameters enable us to distinguish rodrich and plate-rich nematic phases, as the most efficient packing of the particles in homogeneous phases of hard rods and plates requires a perpendicular arrangement of the symmetry axes of the two particles. This means that if the order parameter of the plates is positive, i.e., if they are aligned in the direction of the nematic director, the order parameter of the rods is negative, and vice versa.

\section{STABILITY ANALYSIS}

It has been known for some time that the fluid phases of anisotropic hard particles such as ellipsoids, ${ }^{49,50}$ spherocylinders, ${ }^{51-54}$ and cylinders, ${ }^{8}$ can exhibit varying degrees of orientational and translational order which give rise to liquid crystalline phase behavior including nematic, smec- tic, and columnar phases. It is also known that these phases are stabilized by the reduction of the excluded volume in the aligned configurations. In mixtures of anisotropic hard particles, ordering transitions are also observed, and in addition, phase separation may occur between phases of the same symmetry (isotropic-isotropic, nematic-nematic demixing) if the two components are of a very different size and/or shape. In these cases the demixing transitions are possible when the loss in the entropy of mixing, and of orientational entropy (in the case of a nematic-nematic demixing), are compensated for by a reduction of the excluded volume corresponding to an increase in the free volume (packing) entropy. It is important to note that these are entropy-driven transitions as the interactions are purely repulsive. One should note that isotropic-isotropic demixing is commonly seen in simple fluids (alkane + perfluoroalkane mixtures are a well-known example), ${ }^{55}$ but in these cases the demixing transitions arise due to weak unlike attractive interactions, i.e., the enthalpic terms dominate the free energy of mixing. Another important point is that isotropic demixing is not always observed in mixtures of anisotropic particles because the ordering transitions (leading to stable nematic phases) frequently preempt the separation between the isotropic phases. This competition between demixing and ordering gives rise to a very rich phase behavior, which is the focus of our study. In order to study the competition between the demixing and ordering transitions, we propose a simple approximate procedure to locate the regions of the molecular-parameter space in which isotropic-isotropic phase separation may be stable. Our analysis is based on a comparison of the isotropicnematic bifurcation pressures and the critical pressures of the isotropic-isotropic spinodal.

\section{A. Isotropic-nematic bifurcation}

A first indication of an isotropic-nematic phase transition can be obtained by determining the lowest density at which a nematic solution bifurcates from that of the trivial isotropic solution. It is important to note, however, that the actual transition coincides with the bifurcation point only for second-order (continuous) transitions. In the case of a firstorder isotropic-nematic transition, the bifurcation point merely gives an upper limit for the stability of the isotropic phase.

An isotropic-nematic bifurcation equation can be obtained by studying the limiting behavior of the order parameter $S$ as it approaches zero in the nematic phase. In the case of cylindrically symmetrical hard-particles the excluded volumes are a function of the angle $\gamma$ formed by the symmetry axes of the two molecules, so that $\gamma=\operatorname{arc} \cos \left(\boldsymbol{\omega}_{1} \cdot \boldsymbol{\omega}_{2}\right)$, and the excluded volumes can be written as Legendre polynomials of the form,

$$
v_{i j}(\gamma)=a_{i j}+b_{i j} P_{2}(\cos \gamma)+\cdots,
$$

where $a_{i j}$ and $b_{i j}$ are the zeroth and second order the expansion coefficients. All odd coefficients are zero due to the up-down symmetry of the particles. In our case, the series can be truncated at second order, which corresponds to the leading term of the series. Certain values of the molecular parameters (corresponding to near-spherical particles) would 
promote stable cubatic phases in which the fourth Legendre polynomial is greater than the second; we have not considered these cases here.

After inserting Eq. (14) truncated at second order into the coupled integral equations [Eqs. (11) and (12)], the orientational distribution functions are given by

$f_{1}(\theta)=$

$$
\frac{\exp \left(-\frac{f^{*}(\eta) P_{2}(\cos \theta)}{4\left(x_{1} v_{1}+x_{2} v_{2}\right)}\left(x_{1} b_{11} S_{1}+x_{2} b_{12} S_{2}\right)\right)}{\int \exp \left(-\frac{f^{*}(\eta) P_{2}(\cos \theta)}{4\left(x_{1} v_{1}+x_{2} v_{2}\right)}\left(x_{1} b_{11} S_{1}+x_{2} b_{12} S_{2}\right)\right) d \boldsymbol{\omega}_{1}},
$$

$$
\begin{aligned}
& f_{2}(\theta)= \\
& \quad \frac{\exp \left(-\frac{f^{*}(\eta) P_{2}(\cos \theta)}{4\left(x_{1} v_{1}+x_{2} v_{2}\right)}\left(x_{2} b_{22} S_{2}+x_{1} b_{12} S_{1}\right)\right)}{\int \exp \left(-\frac{f^{*}(\eta) P_{2}(\cos \theta)}{4\left(x_{1} v_{1}+x_{2} v_{2}\right)}\left(x_{2} b_{22} S_{2}+x_{1} b_{12} S_{1}\right)\right) d \boldsymbol{\omega}_{1}},
\end{aligned}
$$

where the addition theorem of spherical harmonics,

$$
\begin{aligned}
P_{2}(\cos \gamma)= & P_{2}\left(\cos \theta_{1}\right) P_{2}\left(\cos \theta_{2}\right) \\
& +2 \sum_{i=1}^{2} \frac{(2-i) !}{(2+i) !} P_{2}^{i}\left(\cos \theta_{1}\right) P_{2}^{i}\left(\cos \theta_{2}\right) \\
& \times \cos \left(i\left(\varphi_{1}-\varphi_{2}\right)\right)
\end{aligned}
$$

has been used, noting that the terms proportional to the associated Legendre polynomials $P_{2}^{i}$ vanish since the nematic phase is uniaxial and does not depend on the azimuthal angle $\varphi$. At the isotropic-nematic bifurcation point the order parameters must be zero. Hence taking the $S_{1} \rightarrow 0$ and $S_{2} \rightarrow 0$ limits in Eqs. (15) and (16), and expanding the exponential function as a Taylor series up to second order $(\exp x \approx 1+x)$ it is possible to write

$$
f_{1}(\theta)=\frac{1-\frac{f^{*}(\eta) P_{2}(\cos \theta)}{4\left(x_{1} v_{1}+x_{2} v_{2}\right)}\left(x_{1} b_{11} S_{1}+x_{2} b_{12} S_{2}\right)}{4 \pi} .
$$

and

$$
f_{2}(\theta)=\frac{1-\frac{f^{*}(\eta) P_{2}(\cos \theta)}{4\left(x_{1} v_{1}+x_{2} v_{2}\right)}\left(x_{2} b_{22} S_{2}+x_{1} b_{12} S_{1}\right)}{4 \pi} .
$$

Using Eq. (13) the corresponding coupled equations for the order parameters are

$$
0=S_{1}+\frac{f^{*}(\eta)}{20\left(x_{1} v_{1}+x_{2} v_{2}\right)}\left(x_{1} b_{11} S_{1}+x_{2} b_{12} S_{2}\right)
$$

and

$$
0=S_{2}+\frac{f^{*}(\eta)}{20\left(x_{1} v_{1}+x_{2} v_{2}\right)}\left(x_{2} b_{22} S_{2}+x_{1} b_{12} S_{1}\right)
$$

The order parameters can be eliminated and a nonlinear equation for the reduced density $\eta^{*}$ is obtained in terms of a function, $y=f^{*}(\eta) /\left[20\left(x_{1} v_{1}+x_{2} v_{2}\right)\right]$ as

$$
0=\left(b_{22} b_{11}-b_{12}^{2}\right) x_{1} x_{2} y^{2}+\left(x_{1} b_{11}+x_{2} b_{22}\right) y+1 .
$$

For each composition the bifurcation reduced density $\eta^{*}$ is obtained numerically from Eq. (22) with a Newton-Raphson method. $^{48}$

\section{B. Spinodal decomposition}

In order to study phase separation in mixtures, the Gibbs free energy is the natural thermodynamic function, and it can be obtained by an appropriate Laplace transformation of the free energy. In reduced form, this can be written as

$$
\widetilde{g}=\tilde{f}+P^{*} / \eta^{*},
$$

where the reduced Gibbs free energy is $\widetilde{g}=\beta G / N$, the reduced Helmholtz free energy $\widetilde{f}=\beta F / N$, and $P^{*}$ $=\eta^{* 2} \partial \tilde{f} / \partial \eta^{*}$ with $P^{*}=\beta P \nu_{1}$.

Thermodynamic stability of a binary mixture with respect to phase separation at given temperature, pressure and composition can be determined from the sign of the second derivative of the Gibbs free energy. If $\left(\partial^{2} \widetilde{g} / \partial x^{2}\right)_{P, T}>0$ the mixture is stable or metastable, while if $\left(\partial^{2} \widetilde{g} / \partial x^{2}\right)_{P, T}<0$ the mixture is unstable. ${ }^{55}$ Therefore the relation

$$
\left(\frac{\partial^{2} \tilde{g}}{\partial x^{2}}\right)_{P, T}=0
$$

represents the limit of stability of the miscible state in a mixture. Equation (24) defines the points of the so-called spinodal curve. We have used the notation $x$ for the mole fraction of one of the components. Unfortunately, the Gibbs free energy cannot be expressed as an explicit function of density. It is more convenient to use the relation,

$$
\left(\frac{\partial^{2} \tilde{g}}{\partial x^{2}}\right)_{P, T}=\left(\frac{\partial^{2} \widetilde{f}}{\partial x^{2}}\right)_{T, \eta^{*}}-\eta^{*} \frac{\left(\frac{\partial^{2} \widetilde{f}}{\partial x \partial \eta^{*}}\right)_{T}^{2}}{\left(2 \frac{\partial \widetilde{f}}{\partial \eta^{*}}+\eta^{*} \frac{\partial^{2} \widetilde{f}}{\partial \eta^{* 2}}\right)_{T, x}}
$$

since the Helmoltz free energy is an explicit function of composition and density. The results of the straightforward but tedious differentiations are not presented here. As for the bifurcation densities, the reduced densities of the spinodal curve are obtained by solving Eq. (24) numerically with the Newton-Raphson method ${ }^{48}$ at each composition.

\section{Stability conditions}

For mixtures of hard anisotropic particles, both the isotropic-nematic and the isotropic-isotropic transitions, while unfavorable in terms of the entropy of mixing, are driven by a reduction of the excluded volumes. In the case of the isotropic-nematic transition, an added gain in free volume is obtained by the alignment of the particles in one of the phases. It is therefore evident that the spinodal decompo- 
sition of the isotropic phase depends strongly on the orientation-dependent terms in the excluded volumes, which give rise to the nematic ordering, since the isotropic-nematic phase transition frequently preempts the isotropic demixing transition. In order to study the competing ordering and demixing effects, it is useful to compare the isotropic-nematic and the spinodal demixing pressures. Having obtained the isotropic-nematic bifurcation reduced density $\eta_{I N}^{*}$ from Eq. (22) and the isotropic-isotropic spinodal reduced density $\eta_{I I}^{*}$ from Eq. (24), the pressures $P^{*}=\beta P \nu_{1}$ can be calculated for a given composition from the following equation for the isotropic phase (which is the same as that of the nematic phase at the bifurcation point),

$$
P^{*}=\eta^{*}\left\{1+\eta^{*} \frac{4-2 \eta\left(x_{1}^{2} a_{11}+2 x_{1} x_{2} a_{12}+x_{2}^{2} a_{22}\right)}{(1-\eta)^{3}}\right\} .
$$

As a good approximation one can reasonably assume that the binary mixture will undergo a phase separation in the isotropic phase if the critical pressure of the spinodal line is below the lowest isotropic-nematic bifurcation pressure. In this way we can define a set of stability conditions for the demixing transitions by determining the minima in the spinodal and bifurcation pressures, and taking them to be equal, so that

$$
\begin{aligned}
& P_{I I}^{*}\left(x_{I I}\right)=P_{I N}^{*}\left(x_{I N}\right), \\
& \left.\frac{\partial P_{I N}^{*}}{\partial x}\right|_{x_{I N}}=0, \\
& \left.\frac{\partial P_{I I}^{*}}{\partial x}\right|_{x_{I I}}=0 .
\end{aligned}
$$

As we will see in the Results section, the spinodal pressurecomposition curve always exhibits a minimum within the composition range $0-1$, but a minimum pressure is not always observed in the isotropic-nematic bifurcation curve (i.e., sometimes the lowest bifurcation pressure corresponds to that of one of the pure components, $x=0$ or $x=1$ ). The second condition in Eq. (27) is not considered if the minimum bifurcation pressure occurs at $x=0$ or $x=1$.

Using Eqs. (22), (24), (26), and (27) the regions of the molecular parameter space in which isotropic demixing is thermodynamically stable can be determined. It is important to recall at this point that these equations do not give the necessary and sufficient conditions to identify the systems possessing the isotropic demixing transition in a rigorous way, since in most cases the isotropic-nematic bifurcation pressures are higher than the isotropic-nematic transition pressures. However, despite the approximate nature of the stability analysis that is proposed, it will become clear from the examples presented in the following sections that the method is extremely useful in giving an indication of the shape of the global phase behavior in complex binary mixtures of hard anisotropic particles. Furthermore, our stability analysis has the advantage of being general. So far no restriction has been imposed as to the shape and size of the particles involved. In the next section we specify the expres- sions for the excluded volume in mixtures of hard cylinders and define the molecular parameters of our study.

\section{MOLECULAR MODELS}

In order to model a mixture of platelike (component 1) and rodlike (component 2) molecules we have used hard cylinders of different aspect ratios, chosen so that one is thin and flat (the plate) and the other long and thin (the rod). The plate molecules have a length $L_{1}$ and diameter $D_{1}$ fixed so that $D_{1} / L_{1}>1$, while the length $L_{2}$ and diameter $D_{2}$ of the rodlike component are set so that $L_{2} / D_{2}>1$. The advantage of modeling both molecules as hard cylinders (as opposed to using ellipsoids, spherocylinders or cut spheres) is that the expression for the excluded volumes are relatively simple. The excluded volumes reduced by the diameter of component $1\left(v_{i j}^{*}=v_{i j} / D_{1}^{3}\right)$ can be written as functions of the aspect ratios $\kappa_{1}=L_{1} / D_{1}$ and $\kappa_{2}=L_{2} / D_{2}$, and the diameter ratio $d=D_{1} / D_{2}$ of the two components, ${ }^{27}$

$$
\begin{aligned}
v_{11}^{*}(\gamma)= & \frac{\pi}{2} \kappa_{1}(1+|\cos \gamma|)+2 \kappa_{1} E(\sin \gamma) \\
& +\left(\frac{\pi}{2}+2 \kappa_{1}^{2}\right) \sin \gamma, \\
v_{22}^{*}(\gamma)= & \left\{\frac{\pi}{2} \kappa_{2}(1+|\cos \gamma|)+2 \kappa_{2} E(\sin \gamma)\right. \\
& \left.+\left(\frac{\pi}{2}+2 \kappa_{2}^{2}\right) \sin \gamma\right\} / d^{3}, \\
v_{12}^{*}(\gamma)= & \frac{\pi}{4}\left(\kappa_{1}+\frac{\kappa_{2}}{d^{3}}\right)+\left(\frac{1+d}{d^{2}}\right)\left(\frac{\pi}{4}+z\right) \sin \gamma \\
& +\left(\frac{\kappa_{1}}{d}+\frac{\kappa_{2}}{d^{2}}\right) E(\sin \gamma)+\frac{\pi}{4}\left(\frac{\kappa_{1}}{d^{2}}+\frac{\kappa_{2}}{d}\right)|\cos \gamma| .
\end{aligned}
$$

Here, $z$ corresponds to the product of the aspect ratios $z$ $=\kappa_{1} \kappa_{2}$, and $E(x)$ is the complete elliptical integral of second kind, defined as $E(x)=\int_{0}^{\pi / 2} \sqrt{1-x^{2} \sin ^{2} \varphi} d \varphi$. As can be seen from Eqs. (28) to (30), the shapes of the particles, given by $\kappa_{1}$, and $\kappa_{2}$, and their relative size $d$, need to be defined in order to determine the excluded volumes. At this stage, however, expressions (28)-(30) are general, and may be used to describe rod-rod, plate-plate or plate-rod mixtures. The value of the parameter $z$ further determines the type of mixture in question. In this work we considered cases with values of $z$ from 1 to 5 , in order to ensure that our models correspond to rod-plate mixtures regardless of the individual aspect ratios. In future, we plan to continue this work by considering molecular parameters corresponding to rod-rod $(z \gg 1)$ and plate-plate mixtures $(z \ll 1)$.

Before concluding this section it is also useful to consider the corresponding excluded volumes for plate and rod particles in the well-known model of Onsager. ${ }^{27}$ In this limit the thickness of the plate particles approaches zero but the diameter is finite so that $\kappa_{1} \rightarrow 0$, while the diameter of the rod 
particles tends to zero compared to their length so that $\kappa_{2}$ $\rightarrow \infty$. Taking these limits in Eqs. (28)-(30), the excluded volumes are given by

$$
\begin{aligned}
& v_{11}^{*}(\gamma)=\frac{\pi}{2} \sin \gamma, \\
& v_{22}^{*}(\gamma)=\frac{2 \kappa_{2}^{2}}{d^{3}} \sin \gamma, \\
& v_{12}^{*}(\gamma)=\frac{\pi \kappa_{2}}{4 d}|\cos \gamma|,
\end{aligned}
$$

where it has also been assumed that the diameter of the plates are much larger than the diameter of the rods ( $d$ $\rightarrow \infty$ ). It is evident that expressions (31)-(33) correspond to the dominant contributions of the total excluded volumes, and hence also determine the phase behavior to a larger extent. However, the lower order contributions (often referred to as end effects) may play a crucial role in mixtures of moderately long and moderately flat particles. In this study we take into account the exact excluded volumes (i.e., including the end effects), but in order to elucidate the differences between the two approaches, we will also examine a system in the Onsager limit.

\section{RESULTS}

As discussed earlier, we aim to determine the regions of the global phase diagram for which isotropic demixing would be stable in mixtures of platelike and rodlike molecules. It is useful to start by solving the stability conditions given in Eq. (27) with the expressions for the excluded volumes given by Eqs. (28)-(30) for different molecular parameters. Throughout this section $x$ refers to the mole fraction of rodlike particles (component 2).

The isotropic-nematic bifurcation curve and the curve for spinodal decomposition in the isotropic phase have been obtained for fixed aspect ratios of $\kappa_{1}=1 / 50$ and $\kappa_{2}=50$, for varying values of the plate-rod diameter ratio $d$ (see Fig. 2). It is noticeable that the critical pressure of the isotropic spinodal decomposition shows a rather unexpected behavior [Fig. 2(a)] for varying diameter ratios: the critical pressure decreases for $d<18$, remains practically constant for $18<d$ $<25$, and later increases for $d>25$. The corresponding critical mole fraction continually increases with increasing diameter ratio. On the other hand, the isotropic-nematic bifurcation pressure always increases with increasing diameter ratio [Fig. 2(b)]. It is also apparent that the minimum bifurcation pressure and its corresponding mole fraction are shifted towards the pure component plate edge of the phase diagram with increasing diameter ratio. For the larger diameter ratios the minimum takes place at $x=0$, which means that the second criteria in Eq. (27) cannot not be used in the stability analysis. In such cases the second criteria is slightly changed by taking the isotropic-nematic bifurcation pressure at $x$ $=0$. From a comparison of Figs. 2(a) and 2(b) one can anticipate that there must be a lower and an upper bound on the stability range of the isotropic demixing transition; note that only one of the two boundaries has previously been proposed in studies of rod-rod mixtures. ${ }^{15,18,36}$
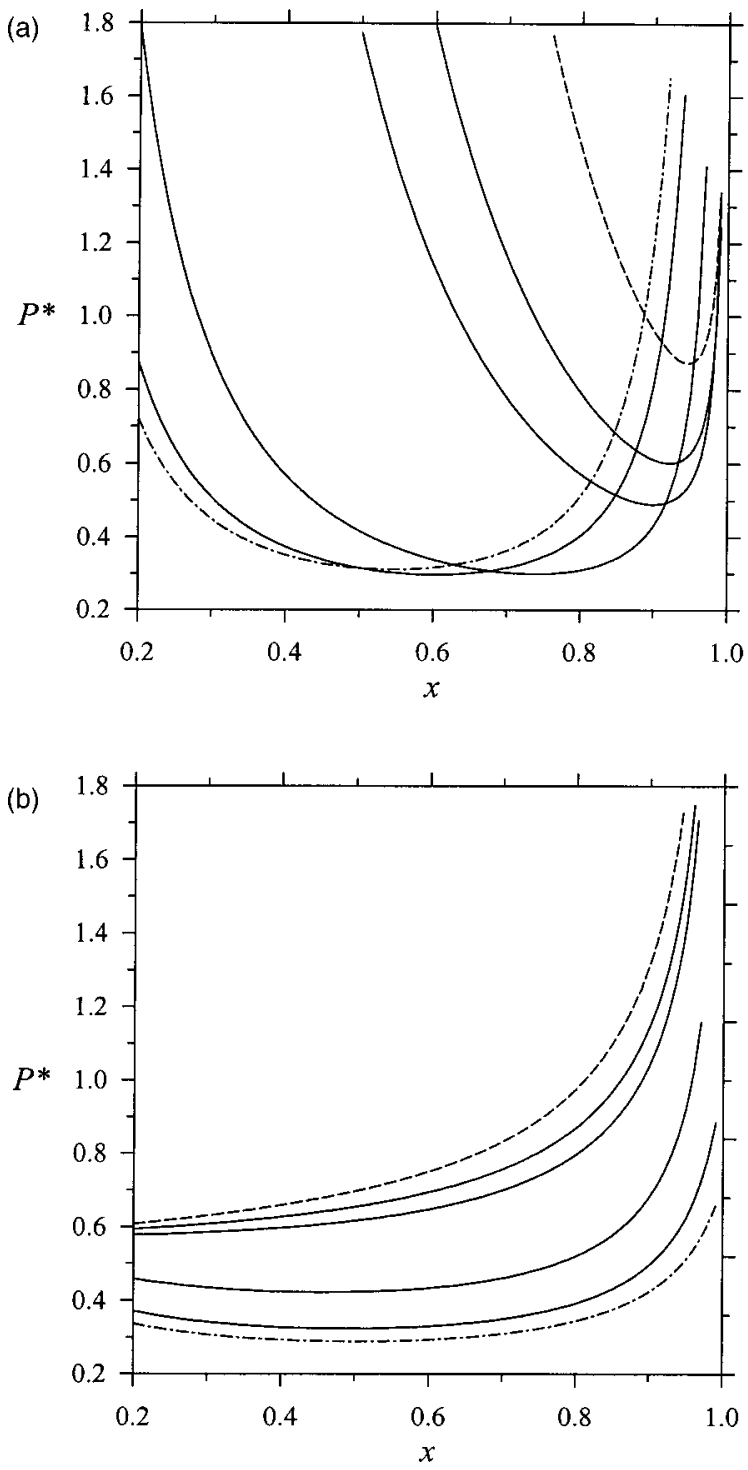

FIG. 2. (a) Spinodal demixing pressures and (b) isotropic-nematic bifurcation pressures for binary mixtures of plates and rods with aspect ratios $\kappa_{1}$ $=1 / 50$ and $\kappa_{2}=50(z=1)$, and varying plate/rod diameter ratio $d$. The values of $d$ which are considered (from bottom to top) are 16 (dasheddotted curve), 18, 25, 50,60, and 80 (dashed curve). $x$ corresponds to the mole fraction of the rods.

The regions of the global parameter space in which isotropic demixing is the thermodynamically stable state can be estimated by solving the stability conditions [Eq. (27)] for a fixed product of the aspect ratios $z$ by varying the aspect ratio of the rods $\kappa_{2}$; the diameter ratios $d$ and compositions $x_{I N}$ and $x_{I I}$ satisfying the conditions for each $\kappa_{2}$ are obtained using a simplex method. ${ }^{48}$ In Fig. 3 the global phase diagram is shown for $z=1$; the boundaries between the stable and metastable isotropic demixing regions are indicated. It is clear that for mixtures involving particles of small aspect ratios (more precisely, $\kappa_{1}<1 / 5.7$ with $\kappa_{2}<5.7$ ) the demixing of the isotropic phase is predicted as the stable thermodynamic state for a very wide range of diameter ratios $d$. One should point out at this stage that our theory might not be accurate in this range of aspect ratios as columnar and cubatic phases, which have not been incorporated in the approach, may be favored. On the other hand, for aspect ratios 


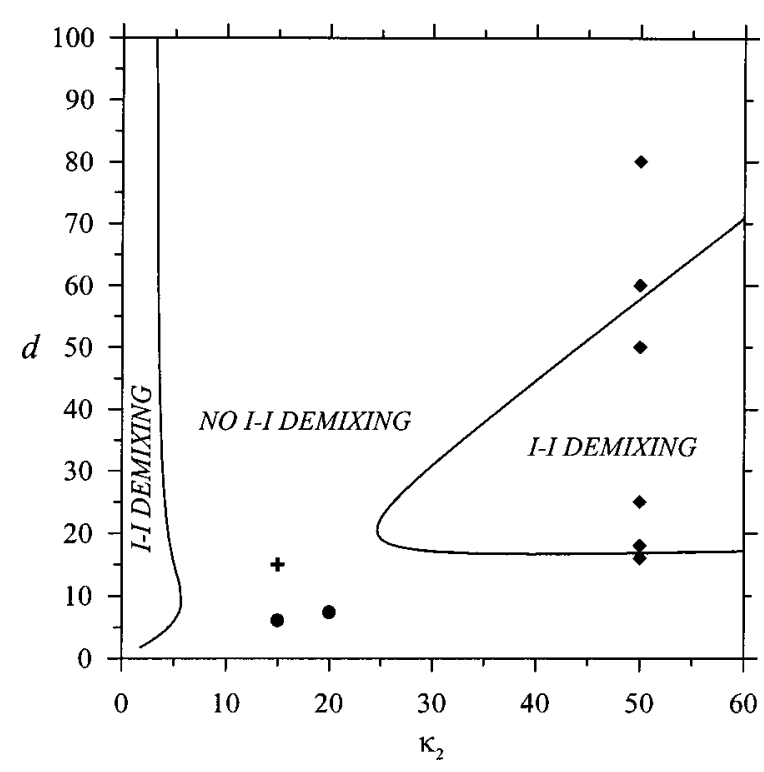

FIG. 3. Global phase diagram for rod-plate mixtures with a product of the aspect ratios of $z=1$. The regions of stable (I-I DEMIXING) and metastable isotropic demixing (NO I-I DEMIXING) are indicated. The continuous curves correspond to the boundaries obtained with the stability analysis proposed in this work. The circles denote the molecular parameters of ellipsoidal plate-rod system studied by Camp et al. (Ref. 44), the cross the molecular parameters of the experimental system of van der Kooij et al. (Ref. 20), and the diamonds the molecular parameters chosen to the test the accuracy of the stability analysis in this work.

of $5.7<\kappa_{2}<24.6$ (with $1 / 5.7<\kappa_{1}<1 / 24.6$ ) isotropic demixing is not observed for any $d$. A second region corresponding to mixtures with stable isotropic demixing is seen for large particle aspect ratios $\left(\kappa_{1}>1 / 24.6\right.$ and $\left.\kappa_{2}>24.6\right)$; in terms of the relative sizes of the particles, the extent of this region increases with increasing aspect ratio. Previously, a number of theoretical ${ }^{56}$ and computer simulation studies ${ }^{43,44}$ of the phase behavior of rod-plate mixtures have been carried out which correspond to mixtures with $z=1$. Demixing of the isotropic phase was not, however, observed in these studies. In agreement with our calculations, when the molecular parameters of Refs. 44 and 56 are plotted in the parameterspace of Fig. 3, they are well within the region in which no isotropic demixing is predicted.

It is important to recall at this stage that the stability conditions that have been used are stipulated as an approximate first indication, and so it is useful to determine explicitly the phase equilibria for a number mixtures in different regions of the global phase diagram presented in Fig. 3. The phase diagrams of six plate-rod mixtures with molecular parameters as indicated in Fig. 3 (diamonds) have been calculated. The molecular aspect ratios are fixed to $\kappa_{1}=1 / 50$ and $\kappa_{2}=50$, while the plate-rod diameter ratio (relative size) is varied from $d=80$ (well above the upper boundary diameter ratio $d=57.79$ ) to $d=16$ (just below the lower boundary diameter ratio $d=16.85)$. The phase diagrams for this series of binary mixtures are collected in Fig. 4. Here the phase behavior is obtained from a full analysis of the Gibbs free energy surface by equating the pressures and chemical potentials of each component in the coexisting phases. In Fig. 4(a) the phase diagram for a plate-rod mixture with $\kappa_{1}$
$=1 / 50, \kappa_{2}=50$, and $d=80$ is shown. The pure fluid of plates $(x=0)$ exhibits an isotropic-nematic transition at $P^{*} \sim 0.4$. Although an ordering (isotropic-nematic) transition is also expected in the pure fluid of rods, this will occur at very high pressures due to the small size of the particles compared to that of the plates. It is noticeable that the addition of rod particles destabilizes the nematic phase of the plates. A reentrant transition in pressure is also observed for rod mole fractions of $x \sim 0.4$, together with a wide isotropic-nematic fractionation for the highest pressures presented; at $P^{*}$ $>0.9$ the isotropic phase coexisting with the plate-rich nematic phase is almost a pure fluid of rods. In agreement with the results of the stability analysis presented in Fig. 3 a demixing of the isotropic phase is not observed in this mixture.

Figures 4(b) and 4(c) correspond to the phase diagrams for mixtures with $\kappa_{1}=1 / 50, \kappa_{2}=50$, and $d=60$ and $d=50$, respectively. In this case a destabilisation of the nematic phase of plates, and re-entrant phase behavior are also seen, but now a region of isotropic-isotropic coexistence is observed in both mixtures. One should recall that the diameter ratio of $d=60$ is just above the upper boundary diameter ratio $d=57.79$ that was obtained using the stability criteria given by expression (27). The consequences of the approximations made in our stability analysis are now apparent: according to the global phase diagram, a mixture with molecular parameters $\kappa_{1}=1 / 50, \kappa_{2}=50$, and $d=60$ would not exhibit isotropic demixing, while a region of isotropicisotropic coexistence, albeit small, is found for this system [cf. Fig. 4(b)]. As can be seen from Fig. 4(c) the two-phase isotropic-isotropic region is broader in composition and pressure for the system with the smaller plate-rod diameter ratio of $d=50$. It is also interesting to note that, for both the system with $d=60$ [Fig. 4(b)] and that with $d=50$ [Fig. 4(c)] the two coexisting isotropic phases are rich in rod particles. The nature of the phase transition is often explained by comparing it to a vapor-liquid transition, ${ }^{10}$ in the sense that the small particles, here the rods (which are also referred to as the depleting agent) give rise to an effective attractive interaction between the larger colloidlike particles, here the plates. For certain relative molecular sizes and concentrations, the interactions induced by the depleting agent result in phase separation. The analogy with the vapor-liquid transition can be made by treating the depleting agent (the small particles) as ideal (although interacting with the colloidal component), and using an appropriate semigrand potential. ${ }^{15,33}$ Unfortunately, this transformation cannot be performed analytically with the Parsons-Lee theory because of the complicated density dependence of the free energy functional due to the incorporation of the interactions between the rodlike particles.

When the plate-rod diameter ratio is decreased to $d$ $=25$ (with $\kappa_{1}=1 / 50$ and $\left.\kappa_{2}=50\right)$, a dramatic change in the phase behavior is observed [see Fig. 4(d)]. In this system the nematic phase of plates is stabilized with respect to the isotropic phase by increasing the mole fractions of rods; an isotropic-nematic azeotropic point is seen at $x=0.34$, and the region of isotropic-isotropic coexistence extends over a wide composition range (although still shifted towards the rodrich region). The phase diagram for the system with a 

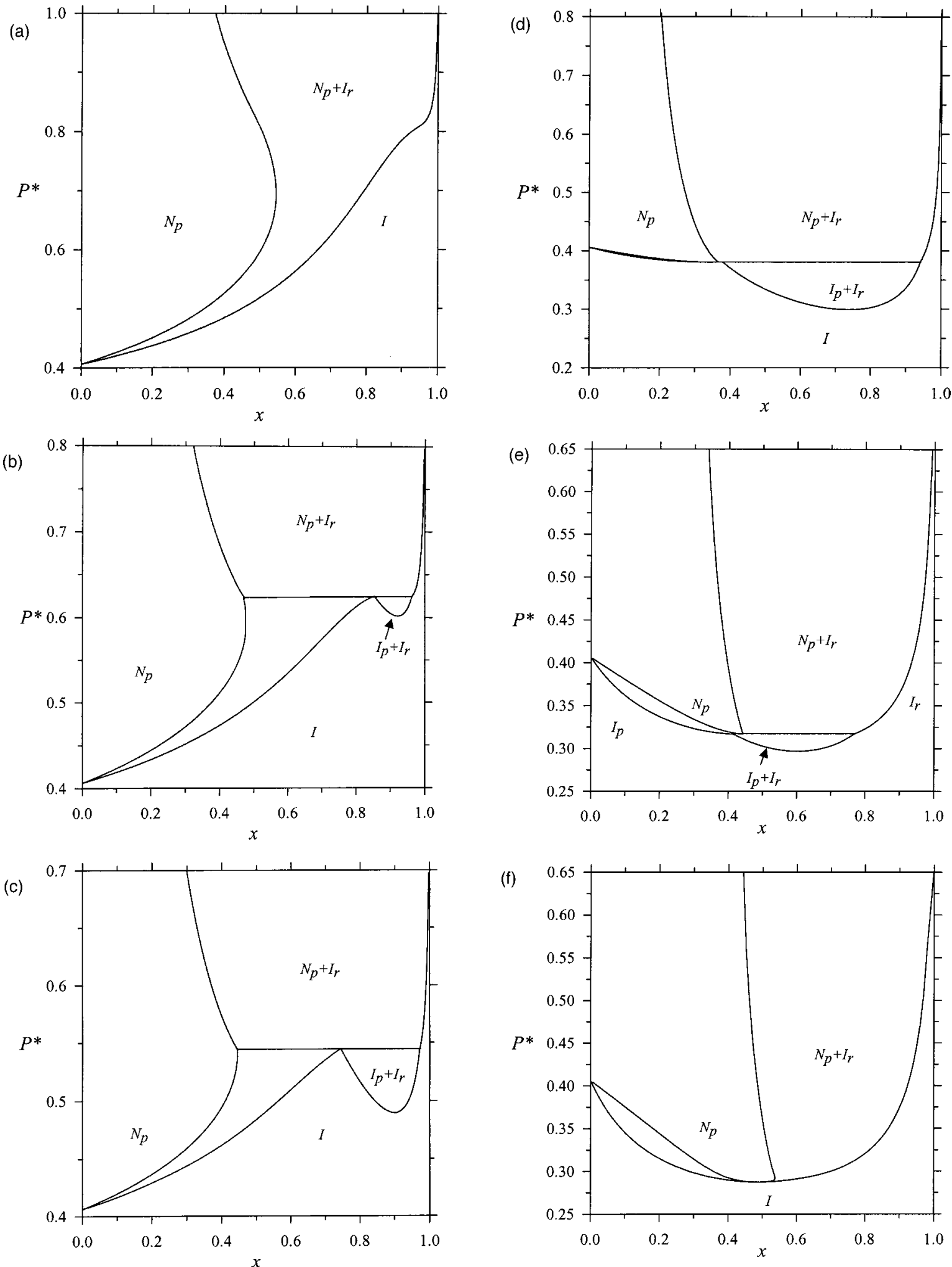

FIG. 4. Pressure-composition $P^{*} x$ representations of the fluid phase diagram for mixtures of plates and rods with aspect ratios of $\kappa_{1}=1 / 50$ and $\kappa_{2}=50(z$ =1) and varying plate/rod diameter ratio $d$ obtained using the Parsons-Lee theory. The values of the corresponding diameter ratios are: (a) $d=80$, (b) $d$ $=60$, (c) $d=50$, (d) $d=25$, (e) $d=18$, and (f) $d=16$. The horizontal lines indicate the nematic-isotropic-isotropic three-phase coexistence pressures for (b) $d=60$, (c) $d=50$, and (d) $d=25$, and the isotropic-nematic-isotropic three-phase coexistence pressure for (e) $d=18$. The labels $I_{r}, I_{p}, N_{r}, N_{p}$ denote the rod-rich isotropic, plate-rich isotropic, rod-rich nematic, and plate-rich nematic phases, respectively. $x$ corresponds to the mole fraction of the rods.

diameter ratio of $d=18$ (just above the lower boundary diameter ratio $d=16.85$ ) is shown in Fig. 4(e). The stabilization of the plate-rich nematic phase is significant in this case. Now a heteroazeotrope is seen,${ }^{55}$ where the coexisting nem- atic phase is at a composition which is between that of the two coexisting isotropic phases. The strong tendency of the mixture to form ordered phases means that isotropic demixing is only observed in a very limited pressure region. The 
fact that the isotropic demixing is still stable is in agreement with the global phase diagram obtained with our stability analysis. For the system with $d=16$ [Fig. 4(f)] further stabilization of the ordered phases means that the isotropic demixing equilibria has become metastable with respect to the isotropic-nematic coexistence over the entire composition range. Another consequence of the lack of isotropicisotropic phase coexistence is that one recovers the isotropic-nematic azeotropic behavior seen for the system with the larger plate-rod diameter ratio [cf. Fig. 4(d)].

It is very gratifying to find that the existence of the upper and lower critical values of the diameter ratio as bounds of stability of isotropic demixing estimated with the simple stability criteria has been confirmed in the case where $z=1$. The predictions of the stability analysis are found to be very reliable for the lower boundary, even though the pressures of the isotropic-nematic transitions are usually lower than the bifurcation pressures. The underestimation of the upper boundary is not surprising as in most of these cases the bifurcation curve does not have a minimum, and instead the bifurcation pressure of the pure plate fluid is used. The minimum in the spinodal curve, however, corresponds to compositions very close to the pure component rods [see Fig. 2(a)], and the isotropic-nematic bifurcation pressure at this composition may be much higher than estimated.

Let us now re-examine a mixture with molecular parameters of $\kappa_{1}=1 / 50, \kappa_{2}=50$, and $d=25$. Wensink et al. ${ }^{56}$ have studied the phase behavior of this mixture using the Onsager second virial theory with the Parsons-Lee scaling, i.e., where the free energy is given by Eq. (1) as in our present work. Two significant approximations are however made in their work: first of all only the leading terms in the excluded volumes are included, so that the end effects are neglected [Eqs. (31)-(33) are used instead of Eqs. (28)-(30)]; secondly, a trial function is used to determine the equilibrium orientational distribution functions instead of the essentiallyexact numerical solution. According to our stability and phase equilibria calculations, the mixture should exhibit isotropic-isotropic demixing [see Figs. 3 and 4(d)]. Wensink et $a .^{56}$ do not however report this demixing. We have already pointed out that the method used to determine the equilibrium orientational distribution functions can have a marked effect on the predicted phase behavior of ordered phases (e.g., see Ref. 25). However, this should not affect the prediction of the phase behavior in isotropic states. Hence, we expect that the observed difference between our results and the phase behavior obtained by Wensink et al. is due to neglecting the lower order terms in the excluded volume. In order to confirm this statement we have performed phase equilibria calculations for the mixture using only the leading terms in the excluded volume [Eqs. (31) -(33)], but with the numerical solution of the orientational distribution functions, and compared it with the results obtained when the exact excluded volumes [Eqs. (28)-(30)] are used. The resulting phase diagrams are compared in Fig. 5. It is clear that the lower-order terms in the excluded volumes are crucial in determining the isotropic demixing transition. When the end effects are taken into account, the isotropic-nematic transition is seen at higher pressures, and at the same time the

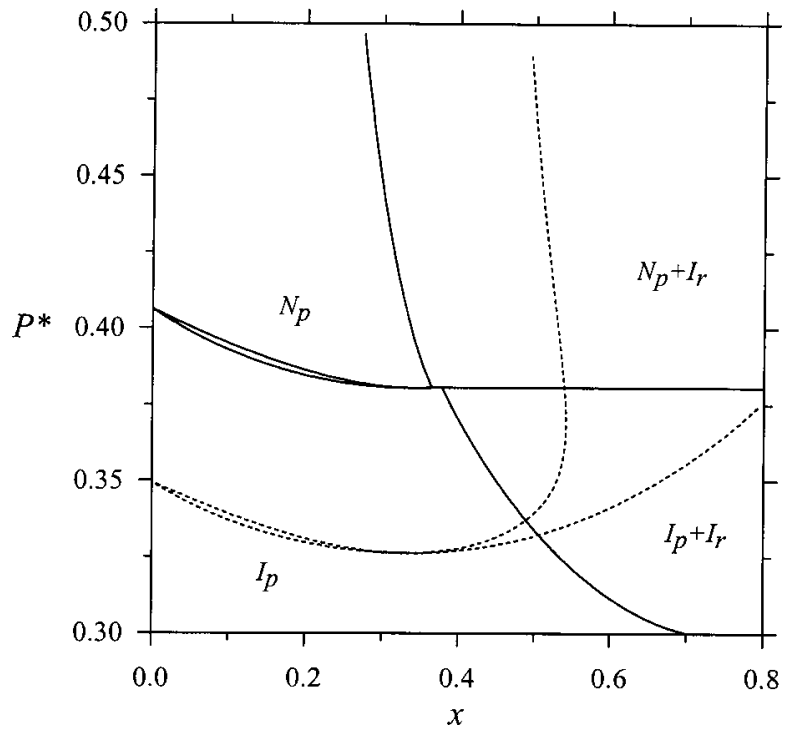

FIG. 5. Pressure-composition $P^{*} x$ phase diagrams for a mixture of plates and rods with aspect ratios of $\kappa_{1}=1 / 50, \kappa_{2}=50$, and plate/rod diameter ratio $d=25$. The continuous curves correspond to the calculations incorporating the complete excluded volume interactions [Eqs. (28)-(30)], while the dashed curves correspond to the calculations using only the leading terms of the excluded volume expressions [Eqs. (31)-(33)].

critical point of the spinodal decomposition is brought down to lower pressures. The lower-order terms in the excluded volume play a significant role even for molecules of reasonably large aspect rations. We have estimated that in the case of a plate-rod symmetric mixture, i.e., a mixture in which the volumes of the two particles are equal (corresponding to a product of aspect ratios of $z=\pi / 4$ ), the end effects become negligible only for aspect ratios of $\kappa_{2}>1000 .^{26}$

We have also investigated the effect of varying the relative shapes of the two components (which translates as varying the parameter $z$, the product of the aspect ratios) on the phase behavior. As mentioned in Sec. II we limit our study to values of $z$ between 1 and 5 to ensure that we are considering plate-rod mixtures and not rod-rod or plate-plate systems. The global phase diagram with $z=1.45$ (see Fig. 6) is rather different to that shown in Fig. 3 which corresponds to $z=1$. In contrast with the phase diagram corresponding to $z=1$ when $z=1.45$, the regions corresponding to mixtures with stable isotropic demixing transitions are connected, so that isotropic demixing can be observed for all $\kappa_{2}>1.8$.

A check of the stability analysis has been carried out for mixtures of particles of moderate elongation $\left(\kappa_{1}=1.45 / 5\right.$ and $\left.\kappa_{2}=5\right)$. In Fig. 7 the phase diagrams of the plate-rod mixture for a plate/rod diameter ratio of $d=6$ (above the boundary diameter ratio $d=4.15$ ) and of $d=4$ (just below the boundary diameter value) are presented. A destabilization of the nematic phase of plates is seen in both systems, together with a strong fractionation between the rod-rich isotropic and plate-rich nematic phases, and re-entrant phenomena in plate-rich composition range. The main difference between the phase behavior of the two systems is, as suggested by the stability analysis, the presence of two coexisting isotropic phases in the mixture with $d=6$.

A further increase in the product of aspect ratios $z$ results 


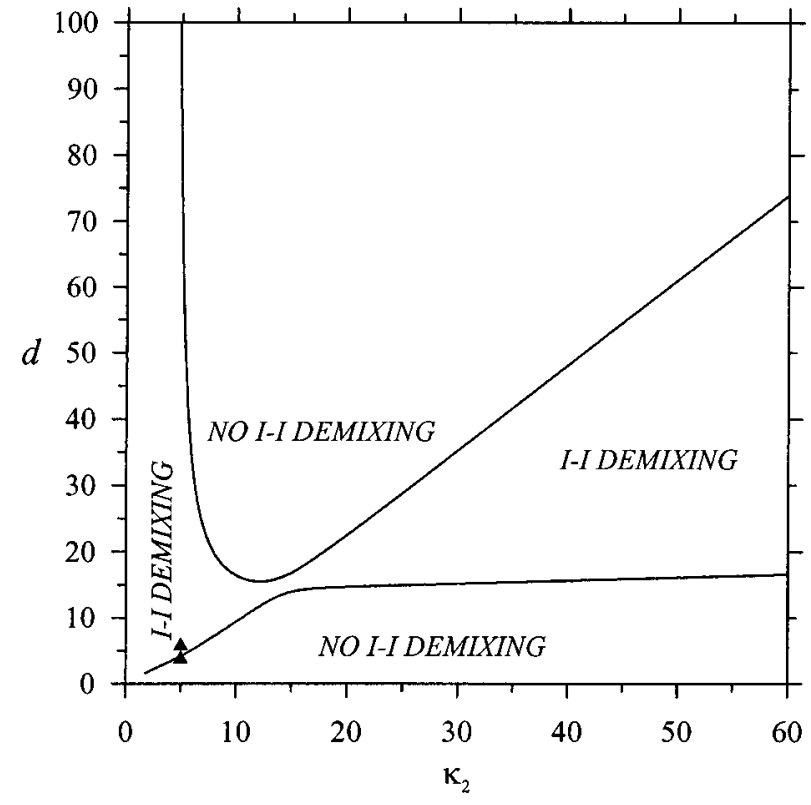

FIG. 6. Global phase diagram for mixtures of plates and rods with a product of the aspect ratios of $z=1.45$ indicating the regions of isotropic demixing. The continuous curves correspond to the boundaries obtained with the stability analysis proposed in this work. The triangles indicate the molecular parameters of the systems studied (see also Fig. 7).

in a greater stabilization of isotropic demixing, as shown for $z=2.5$ in Fig. 8. The single region of the global phase diagram corresponding to mixtures with isotropic demixing already seen for $z=1.45$ (see Fig. 5) is much wider for the system with $z=2.5$ as can be seen from Fig. 8. The value of the lower boundary plate/rod diameter ratio increases for increasing aspect ratios of the rod particles $\left(\kappa_{2}\right)$ up to at least values of $\kappa_{2}=60$, while the upper boundary exhibits a minimum diameter ratio of $d=34.35$ at $\kappa_{2}=17$. Recently, van der Kooij and Lekkerkerker ${ }^{20}$ have carried out an experimental study exploring the phase diagram of a colloidal system of rod and platelike particles. They use platelike gibbsite $\left(\mathrm{Al}(\mathrm{OH})_{3}\right)$ and rodlike bohemite $(\mathrm{AlOOH})$ colloidal particles, which are rigid and essentially behave like hard particles. In their system, the volumes of the plates are much bigger than the volumes of the rods, as in our study. However, the experimental system is highly polydisperse, which gives rise to an extremely rich phase diagram. Together with isotropic-nematic and nematic-nematic coexistence, four and five-phase coexistence are also observed, unfortunately the experimental system does not exhibit isotropic-isotropic phase separation. We have used our analysis to investigate the possibility of observing this phase behavior with the types of particles examined by Lekkerkerker et al. ${ }^{20}$ The average aspect ratio of the rods is $\kappa_{2}=10$, while the polydispersity of the platelike particles gives a range for the product of the aspect ratios $z$ between 0.5 and 3 . In principle by purifying the mixture it may be possible to study a system of molecular parameters $\kappa_{2}=10, \kappa_{1}=2.5 / 10$ and $d=10$, which may have an extensive region of isotropic-isotropic phase separation (see Fig. 9). It is clear that the demixing transition would take place over a wide range of pressures and compositions, and above the three-phase pressure, a nematic phase
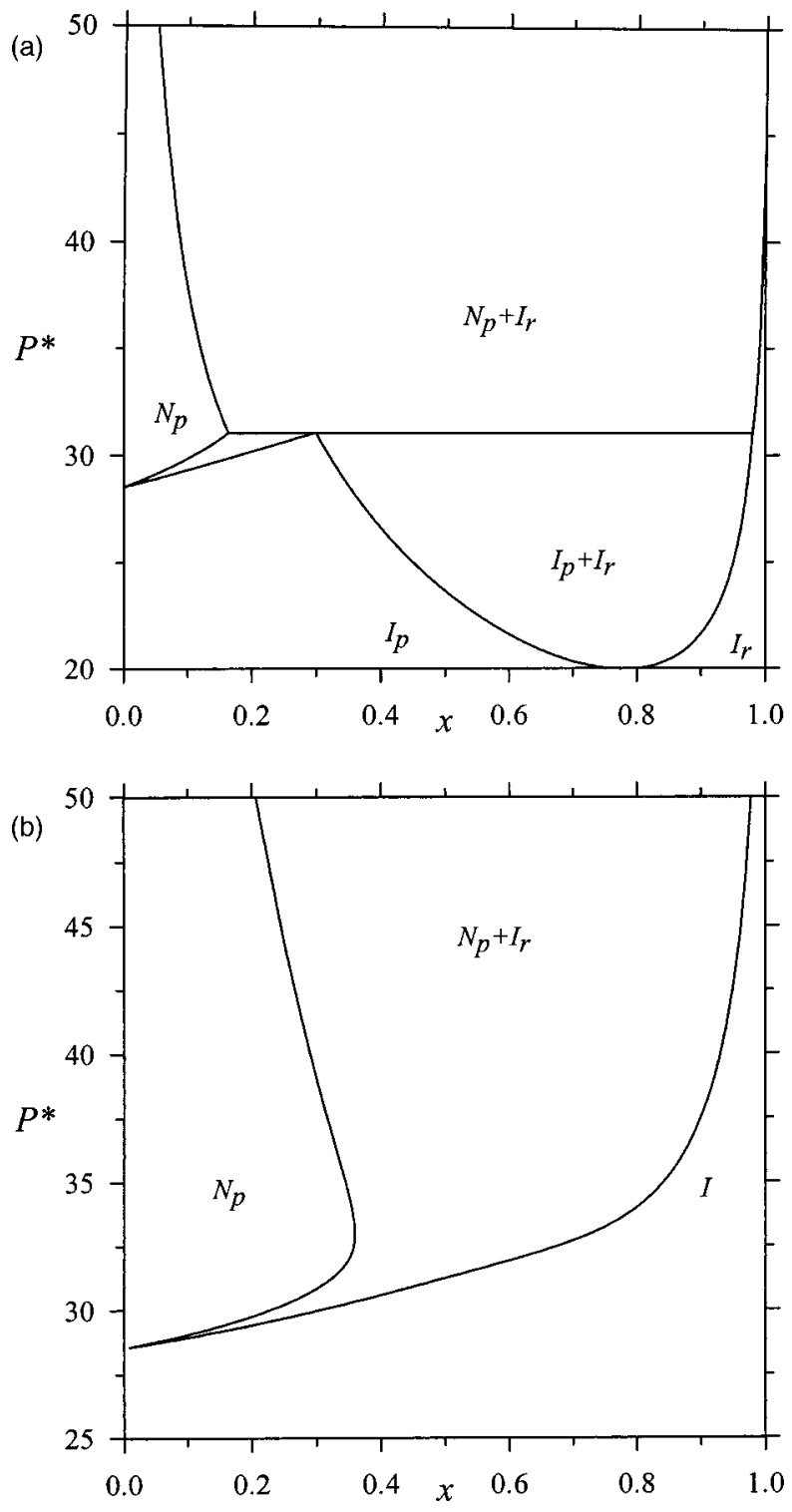

FIG. 7. Pressure-composition $P^{*} x$ representation of the phase diagram for mixtures of plates and rods with aspects ratios $\kappa_{1}=1.45 / 5$ and $\kappa_{2}=5(z$ $=1.45$ ) obtained using the Parsons-Lee theory for plate/rod diameter ratios of (a) $d=6$ and (b) $d=4$. The horizontal line in (a) indicates the nematicisotropic-isotropic three-phase coexistence pressure. See Fig. 4 for more details of the notation.

of plates is found in coexistence with an isotropic phase which is practically pure in rods. From an experimental point of view, a density-density representation of the phase diagram is usually more convenient [Fig. 9(b)], although it should be noted that in this representation the tie lines which connect the coexisting phases are not horizontal. The packing fraction of the plates $\eta_{p}$ and rods $\eta_{r}$ are obtained from the total packing fraction as $\eta_{p}=(1-x) \eta^{*}$, and $\eta_{r}$ $=x \eta^{*} v_{2} / v_{1}$. It may be noted that the coexisting packing fractions of the isotropic and nematic phases close to the pure plate-fluid are very high $(50 \%-60 \%)$, which may suggest that more ordered columnar or solid phases would be stable instead of the nematic phase examined here. The critical point of the isotropic-isotropic transition corresponds to a packing fraction of about $35 \%$. 


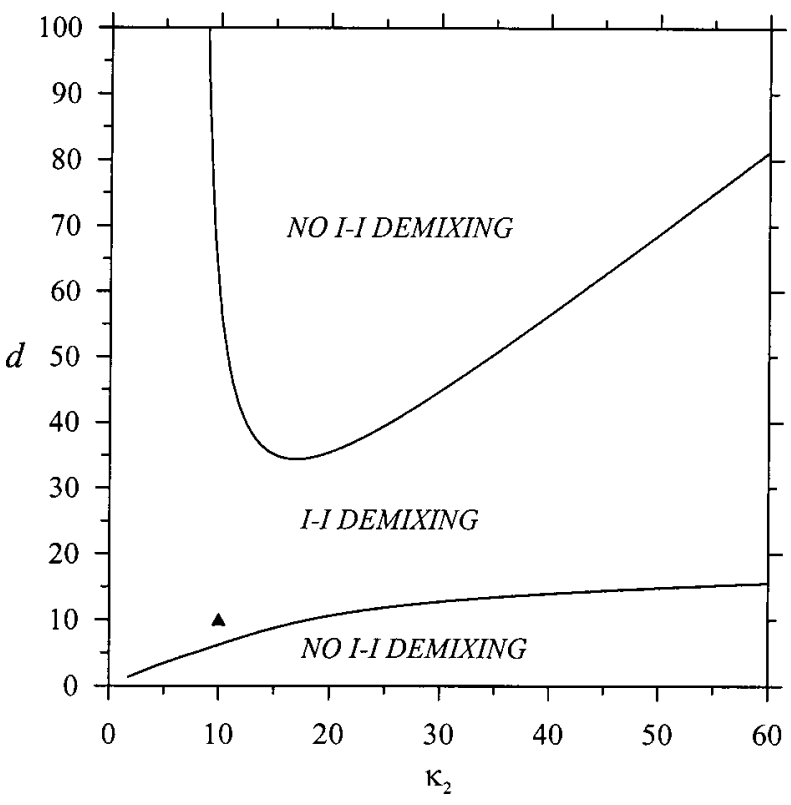

FIG. 8. Global phase diagram for mixtures of plates and rods with a product of the aspect ratios of $z=2.5$ indicating the regions of isotropic demixing. The continuous curves correspond to the boundaries obtained with the stability analysis proposed in this work. The triangle indicates the molecular parameters of the systems studied (see also Fig. 9).

In Fig. 10 we summarize all of our calculations in two global phase diagrams in which the regions corresponding to mixtures with isotropic demixing are indicated for varying plate/rod diameter ratio $d$ [Fig. 10(a)], and volume ratio [Fig. $10(\mathrm{~b})]$. When the product of aspect ratios is $z=1$ two separate regions are observed, one for moderately elongated rodlike particles and the other for long rodlike particles. On increasing $z$ the two regions get closer, and eventually meet for the system with $z=1.428$. A continuous region of isotropic demixing is seen for $z>1.428$; the region widens for increasing $z$. We have only considered cases up to $z=5$ to ensure that our calculations model the phase behavior of rodlike and platelike mixtures. The molecular parameters corresponding to the mixture studied by van der Kooij and Lekkerkerker ${ }^{20}$ are indicated in [Fig. 10(a)]. It is interesting to note that the possibility of observing isotropic-isotropic coexistence is determined by the product of the aspect ratios of the two components (our parameter $z$ ). Our calculations predict that for mixtures with $z>1.5$ isotropic demixing can be expected in the experimental system.

\section{CONCLUSIONS}

In this contribution we have considered the molecular requirements for the stabilization of an isotropic demixing transition in mixtures of plate- and rodlike particles. Based on a comparison of the critical pressure of the isotropic demixing transition and the lowest pressure of the isotropicnematic bifurcation curve, a global phase diagram in terms of the molecular parameters has been obtained which indicates the regions with and without isotropic demixing. The stability analysis that we propose is very simple but it is also approximate, as the isotropic-nematic bifurcation pressure rarely coincides with the coexistence pressure in first-order (a)

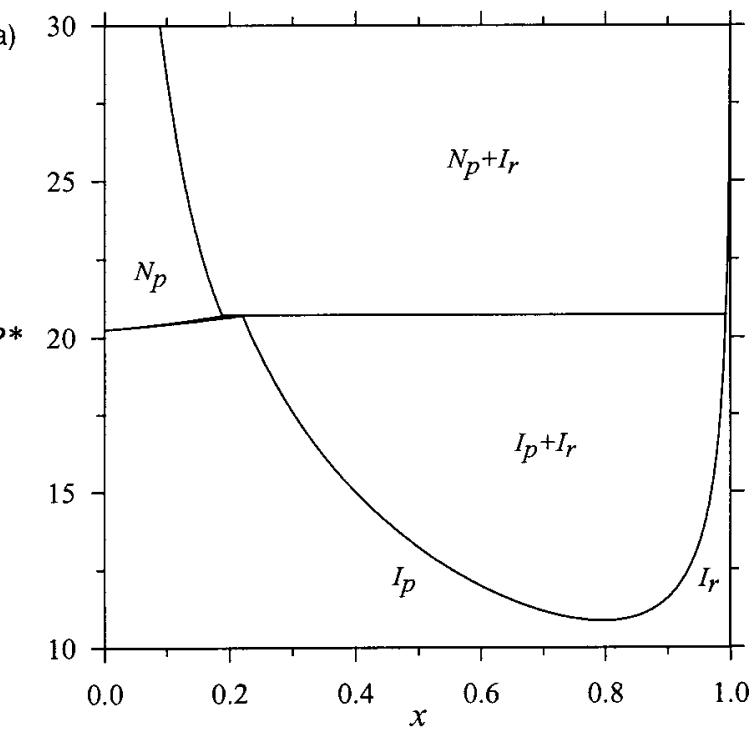

(b)

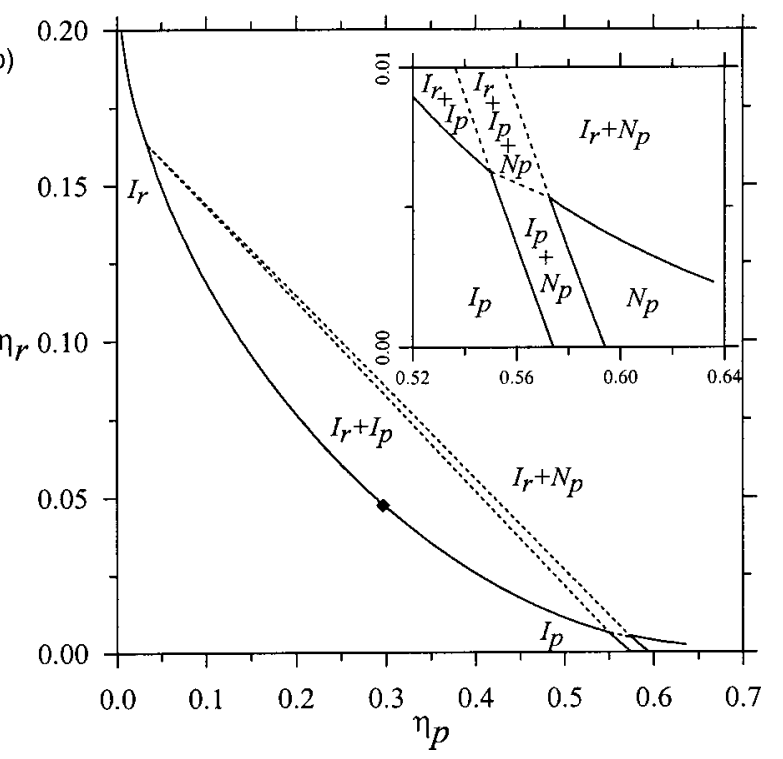

FIG. 9. (a) Pressure-composition $P^{*} x$ representation of the phase diagram of a mixture of plates and rods with aspect ratios of $\kappa_{1}=2.5 / 10$ and $\kappa_{2}$ $=10(z=2.5)$ obtained using the Parsons-Lee theory for a plate/rod diameter ratio of $d=10$. The horizontal lines indicate the nematic-isotropicisotropic three-phase pressure. (b) Phase diagram of the system in the density-density $\left(\eta_{r}-\eta_{p}\right)$ representation. The diamond shows the location of the critical point of the isotropic phase separation. The three phase region is highlighted in the figure inset. See Fig. 4 for more details of the notation.

transitions. Moreover, the extent of the isotropic-nematic fractionation, which no doubt also determines the stability of the isotropic-isotropic demixing transition, cannot be predicted with the bifurcation analysis. This having been said, we have shown that our simple method gives a very good estimate of the molecular parameters for which isotropic demixing is stable.

Two important conditions have also been imposed in our present study. The first is that we have focused on platelike molecules which are of much greater volume than the rodlike particles. This means that the plates play the role of colloidal particles while the rods mediate a colloidal "vapor-liquid" transition. It has already been observed in a number of studies $^{33,35}$ that a diameter ratio which is different than 1 is a 

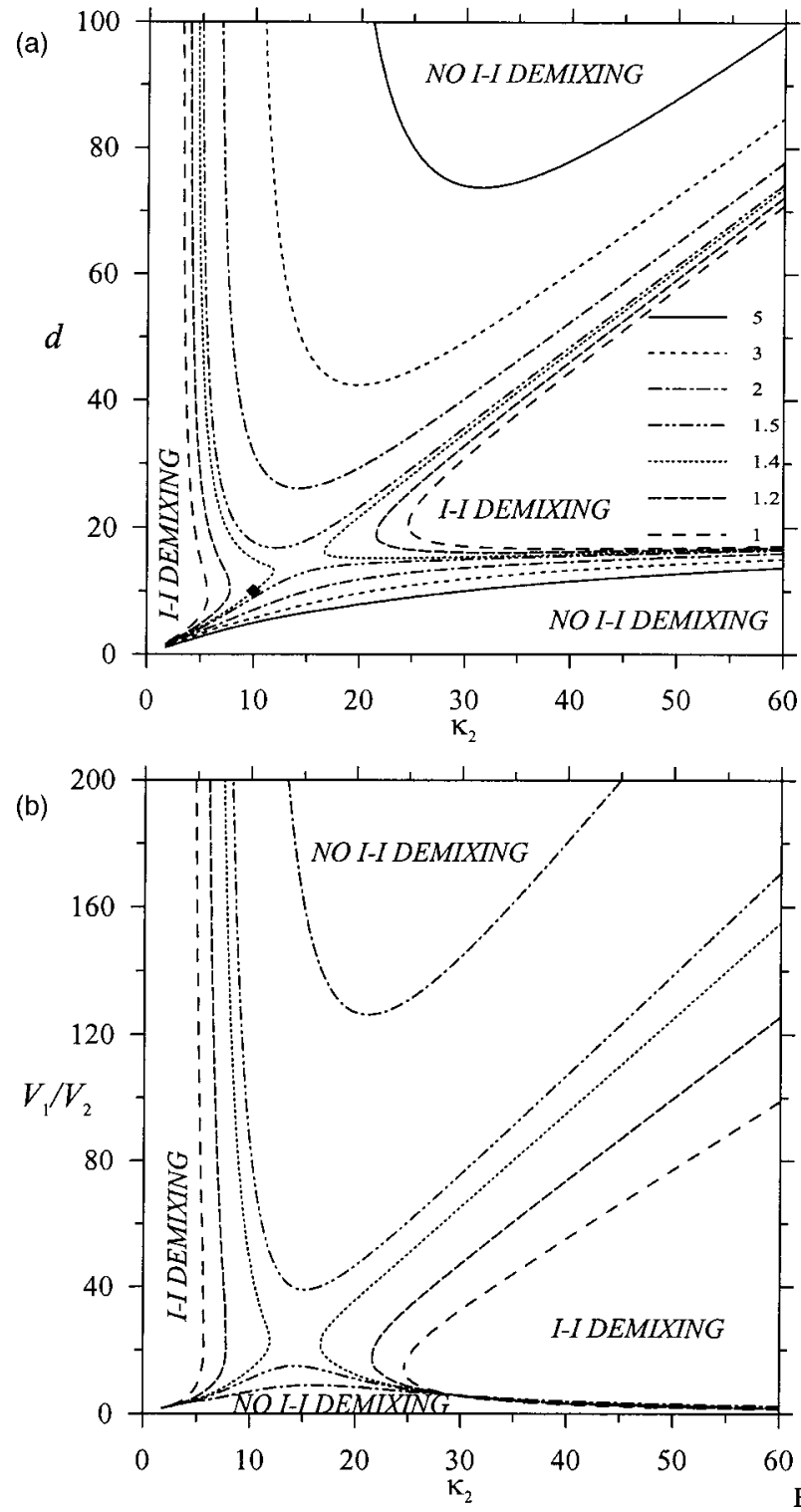

FIG. 10. Global phase diagrams for rod-plate mixtures for varying $z$ indicating the regions of isotropic demixing. The curves correspond to the boundaries obtained with the stability analysis proposed in this work. (a) Diameter ratio-aspect ratio plane, and (b) volume ratio-aspect ratio plane. The values of $z$ considered are $1,1.2,1.4,1.5,2,3$, and 5 . The diamond denotes the experimentally system studied by Lekkerkerker et al. (Ref. 20).

necessary condition for the stabilization of isotropicisotropic transitions in hard-particle systems. This condition is important, but not sufficient, for fixed-shape systems (such as our cylinder-cylinder mixture); the depletion potential between the colloidal (bigger) particles, which is due to the presence of smaller particles, has a critical value beyond which isotropic phase separation can be induced. If the rodlike particles are too small, an ordering (isotropic-nematic) transition preempts the isotropic demixing. Second, it should also be noted that our calculations are not suited for aspect ratios close to 1 , because more ordered phases such as cubatic, columnar, and solid phases, may be favored for these aspect ratios.

As well as isotropic demixing, a variation of the molecular parameters results in the stabilization or destabilization of the nematic-isotropic transition, sometimes associated with a marked fractionation of composition, reentrant phenomena, and azeotropic points. A common trend is seen in our results, which indicates that low rod/plate volume ratios $\left(V_{2} / V_{1}\right)$ are associated with a destabilization of the nematic phase with respect to the isotropic phase.

We have also considered a system with molecular parameters close to those of systems examined in recent experimental studies of rod-plate polydisperse mixtures. ${ }^{4,20}$ Our aim is to provide a guide for future experiments to help locate regions of isotropic-isotropic demixing; our calculations indicate that this may be achieved by reducing the polydispersity of the experimental system. It is likely that the longer rods $\left(\kappa_{2}>10\right)$ will promote the phase transition, but the aspect ratio of the plates is also important since systems with higher values of the product of aspect ratios $(z)$ are associated with extensive isotropic-isotropic demixing regions. We hope that our predictions will also be of interest in future theoretical and simulation studies concerned with the behavior of complex fluids.

\section{ACKNOWLEDGMENTS}

S.V. would like to thank the ROPA program of the Engineering and Physical Sciences Research Council (EPSRC) for a research fellowship (GR/N03358), and A.G. would like to thank the EPSRC for the award of an Advanced Research Fellowship. We acknowledge support from the Joint Research Equipment Initiative (JREI) of the EPSRC for computer hardware (GR/M94427), and the Royal SocietyWolfson Foundation for the award of a refurbishment grant.

${ }^{1}$ P. B. Warren, Europhys. Lett. 46, 295 (1999).

${ }^{2}$ R. P. Sear, Phys. Rev. Lett. 82, 4244 (1999).

${ }^{3}$ H. Zocher, Anorg., Allg. Chem. 147, 91 (1925).

${ }^{4}$ F. M. van der Kooij and H. N. W. Lekkerkerker, Langmuir 16, 10144 (2000).

${ }^{5}$ F. M. van der Kooij and H. N. W. Lekkerkerker, J. Phys. Chem. B 102, 7829 (1998)

${ }^{6}$ F. M. van der Kooij, K. Kassapidou, and H. N. W. Lekkerkerker, Nature (London) 406, 868 (2000).

${ }^{7}$ A. B. D. Brown, S. M. Clarke, and A. R. Rennie, Langmuir 14, 3129 (1998).

${ }^{8}$ J. A. C. Veerman and D. Frenkel, Phys. Rev. A 45, 5632 (1992); R. Blaak, D. Frenkel, and B. M. Mulder, J. Chem. Phys. 110, 11652 (1999).

${ }^{9}$ X. You, Ph.D. thesis, University of Manchester, 2002.

${ }^{10}$ H. N. W. Lekkerkerker, P. Buinning, J. Buttenhuis, G. J. Vroege, and A. Stroobants, in Observation, Prediction, and Simulation of Phase Transitions in Complex Fluids, NATO Series, edited by M. Baus, L. F. Rull, and J.-P. Ryckaert (1995), Vol. 460.

${ }^{11}$ F. M. van der Kooij, M. Vogel, and H. N. W. Lekkerkerker, Phys. Rev. E 62, 5397 (2000)

${ }^{12}$ J. Buitnehuis, L. N. Donselaar, P. A. Buining, A. Stroobants, and H. N. W. Lekkerkerker, J. Colloid Interface Sci. 175, 46 (1995).

${ }^{13}$ Z. Dogic and S. Fraden, Philos. Trans. R. Soc. London, Ser. A 359, 997 (2001)

${ }^{14}$ M. Adams, Z. Dogic, S. L. Keller, and S. Fraden, Nature (London) 393, 349 (1998).

${ }^{15}$ R. Sear and G. Jackson, J. Chem. Phys. 103, 8684 (1995).

${ }^{16}$ P. G. Bolhuis, A. Stroobants, D. Frenkel, and H. N. W. Lekkerkerker, J. Chem. Phys. 107, 1551 (1997).

${ }^{17}$ G. A. Vliegenhart and H. N. W. Lekkerkerker, J. Chem. Phys. 111, 4153 (1999).

${ }^{18}$ P. C. Hemmer and T. H. Marthinsen, Mol. Phys. 100, 667 (2002).

${ }^{19}$ S. Zhang, P. A. Reynolds, and J. S. van Duijneveldt, J. Chem. Phys. (submitted). 
${ }^{20}$ F. M. van der Krooij and H. N. W. Lekkerkerker, Phys. Rev. Lett. 84, 781 (2000).

${ }^{21}$ R. Alben, J. Chem. Phys. 59, 4299 (1973).

${ }^{22}$ A. G. Vanakaras and D. J. Photinos, Mol. Cryst. Liq. Cryst. 299, 65 (1997).

${ }^{23}$ A. Chrzanowska, Phys. Rev. E 58, 3229 (1998).

${ }^{24}$ E. P. Sokolova, I. K. Tokhadze, and N. A. Smirnova, Russ. J. Phys. Chem. 75, 1319 (2001).

${ }^{25}$ S. Varga, A. Galindo, and G. Jackson, Phys. Rev. E 66, 011707 (2002).

${ }^{26}$ S. Varga, A. Galindo, and G. Jackson, J. Chem. Phys. (accepted).

${ }^{27}$ L. Onsager, Ann. N.Y. Acad. Sci. 51, 627 (1949).

${ }^{28}$ H. N. W. Lekkerkerker, Ph. Coulon, R. Van Der Haegen, and R. Deblieck, J. Chem. Phys. 80, 3427 (1984).

${ }^{29}$ T. M. Birshtein, B. I. Kogelov, and V. A. Pruamitsyn, Polym. Sci. U.S.S.R. 30, 316 (1988).

${ }^{30}$ R. van Roij, B. M. Mulder, and M. Dijkstra, Physica A 261, 347 (1998).

${ }^{31}$ S. Varga and I. Szalai, Phys. Chem. Chem. Phys. 2, 1955 (2000).

${ }^{32}$ H. H. Wensink, G. J. Vroege, and H. N. W. Lekkerkerker, J. Phys. Chem. B 105, 10610 (2001).

${ }^{33}$ R. van Roij and B. Mulder, Phys. Rev. E 54, 6430 (1996).

${ }^{34}$ M. Dijkstra and R. van Roij, Phys. Rev. E 56, 5594 (1997).

${ }^{35}$ P. C. Hemmer, J. Stat. Phys. 100, 3 (2000).

${ }^{36}$ H. Bosetti and A. Perera, Phys. Rev. E 63, 021206 (2001).

${ }^{37}$ S. Asakura and F. Oosawa, J. Chem. Phys. 22, 1255 (1954).

${ }^{38}$ R. Zwanzig, J. Chem. Phys. 39, 1714 (1963).

${ }^{39}$ M. A. Bates and D. Frenkel, Phys. Rev. E 62, 5225 (2000).

${ }^{40}$ S. Zhang, P. A. Reynolds, and J. S. van Duijneveldt, Mol. Phys. 100, 3041 (2002).
${ }^{41}$ J. D. Parsons, Phys. Rev. A 19, 1225 (1979).

${ }^{42}$ S. D. Lee, J. Chem. Phys. 87, 4972 (1987).

${ }^{43}$ P. J. Camp and M. P. Allen, Physica A 229, 410 (1996).

${ }^{44}$ P. J. Camp, M. P. Allen, P. G. Bolhuis, and D. Frenkel, J. Chem. Phys. 106, 9270 (1997).

${ }^{45}$ F. Carnahan and E. Starling, J. Chem. Phys. 51, 635 (1969); J.-P. Hansen and I. R. McDonald, Theory of Simple Liquids, 2nd ed. (Academic, London, 1986).

${ }^{46}$ T. Boublik, J. Chem. Phys. 53, 471 (1970).

${ }^{47}$ G. A. Mansoori, N. F. Carnahan, K. E. Starling, and T. W. Leland, J. Chem. Phys. 54, 1523 (1971)

${ }^{48}$ W. H. Press, B. P. Flannery, S. A. Teukolsky, and W. T. Vetterling, $N u-$ merical Recipes in Fortran, 1st ed. (Cambridge University Press, Cambridge, 1986).

${ }^{49}$ D. Frenkel and B. M. Mulder, Mol. Phys. 55, 1171 (1985).

${ }^{50}$ M. P. Allen and C. P. Mason, Mol. Phys. 86, 467 (1995).

${ }^{51}$ D. Frenkel, J. Phys. Chem. 92, 3280 (1988).

${ }^{52}$ D. Frenkel, H. N. W. Lekkerkerker, and A. Stroobants, Nature (London) 332, 822 (1988).

${ }^{53}$ S. C. McGrother, D. C. Williamson, and G. Jackson, J. Chem. Phys. 104, 6755 (1996).

${ }^{54}$ P. Bolhuis and D. Frenkel, J. Chem. Phys. 106, 666 (1997).

${ }^{55}$ J. S. Rowlinson and F. L. Swinton, Liquids and Liquid Mixtures, 3rd ed. (Butterworth Scientific, London, 1982).

${ }^{56}$ H. H. Wensink, G. J. Vroege, and H. N. W. Lekkerkerker, J. Chem. Phys. 115, 7319 (2001). 